\title{
PROXIMAL RELATIONS IN TOPOLOGICAL DYNAMICS ${ }^{1}$
}

\author{
T. S. WU
}

In this note we shall prove that when the proximal relation of a transformation group $(X, T, \pi)$ with compact phase space $X$ is transitive (i.e., it is an equivalence relation), then it is equivalent with the syndetically proximal relation. This would answer two questions in [4, Remark 5].

Standing notations. Let $(X, T, \pi)$ be a transformation group with compact phase space. The proximal relation of $(X, T, \pi)$ is denoted by $P(X)$ and the syndetically proximal relation by $L(X)$. The product transformation group induced by $(X, T, \pi)_{0}$ will be denoted by $(X \times X, T, \rho)$, which is defined by $(x, y) \rho^{t}=\left(x \pi^{t}, y \pi^{t}\right)$ for $(x, y)$ $\in X \times X$ and $t \in T$. For simplicity we shall write $x t$ for $x \pi^{t}$ and $(x t, y t)$ $=(x, y) t$ for $(x, y) \rho^{t}$.

Reference. The proximal relation was studied in [1], [2], [3], [4]. The syndetically proximal relation was defined and studied in [4].

Proposition. If $P(X)$ is transitive, then $P(X)=L(X)$.

Proof. Since $P(X)$ is transitive, so is $P(X \times X)$ [1]. Then each orbit closure $\mathrm{Cl}(x, y) T$ in $(X \times X, T, \rho)$ contains a unique minimal set. Let $(x, y) \in P(X)$. If $\mathrm{Cl}(x, y) T-P(X) \neq \square$, then there is $(a, b)$ $\in \mathrm{Cl}(x, y) T-P(X)$. Let $M$ be the (unique) minimal set contained in $\operatorname{Cl}(a, b) T$. There are two cases.

Case 1. $M \cap P(X)=\square$. By Lemma 2 of [1], there is a point $(u, v) \in M$ such that $((x, y),(u, v)) \in P(X \times X)$. This shows that $(x, u) \in P(X)$, $(y, v) \in P(X)$, a fortiori, $(u, v) \in P(X)$ by the transitivity of $P(X)$. We have the contradiction.

Case 2. $M \cap P(X) \neq \square$. By the definition of $P(X)$, if $\left(x^{\prime}, y^{\prime}\right) \in P(X)$ and $N$ is the minimal set contained in $\mathrm{Cl}\left(x^{\prime}, y^{\prime}\right) T$, then $N \subset \Delta(X)$, the diagonal of $X \times X$. This shows that $M \subset \Delta(X)$, a fortiori, $(a, b)$ $\in P(X)$. We have the contradiction also. Hence, $\mathrm{Cl}(x, y) T \subset P(X)$ when $(x, y) \in P(X)$. By Lemma 5 of $[4], P(X) \subset L(X), P(X)=L(X)$.

CoRollary. $P(X)$ is an equivalence relation if and only if $P(X \times X)$ $=P(X) \times P(X)$.

Received by the editors February 10, 1964.

1 This work was supported by NSF Contract No. G-20301 


\title{
REFERENCES
}

1. J. Auslander, On the proximal relation in topological dynamics, Proc. Amer. Math. Soc. 11 (1960), 890-895.

2. R. Ellis, $A$ semigroup associated with a transformation group, Trans. Amer. Math. Soc. 94 (1960), 272-281.

3. R. Ellis and W. Gottschalk, Homomorphisms of transformation groups, ibid., 94 (1960), 258-271.

4. J. P. Clay, Proximity relations in transformation groups, Trans. Amer. Math. Soc. 108 (1963), 88-96.

STANFORD UNIVERSITY

\section{A TOTALLY BOUNDED, COMPLETE UNIFORM SPACE IS COMPACT}

\author{
D. L. FRANK
}

Let $X$ be a set and $U$ a uniformity on $X$. We will show that if $(X, U)$ is totally bounded, every net in $X$ has a Cauchy subnet. For each $d \in U$, let $S_{d}^{1}, \cdots, S_{d}^{n}$ be a finite covering of $X$ by $d$-spheres. Let $T_{d}$ be the topology on $X$ having $S_{d}^{1}, \cdots, S_{d}^{n}$ as its subbasis. Clearly the space $\left(X, T_{d}\right)$ is compact. Therefore, $Y=\prod_{d \in U}\left(X, T_{d}\right)$ is compact.

Now, let $\left(p_{i}\right)$ be a net in $X$. Then $\Delta \circ\left(p_{i}\right)$ is a net in $Y$, where $\Delta: X \rightarrow Y$ is the diagonal. By compactness, there exists a convergent subnet, $\left(q_{j}\right)$, of $\Delta \circ\left(p_{i}\right)$. Then $\Delta^{-1} \circ\left(q_{j}\right)$ is a subnet of $\left(p_{i}\right)$ which is clearly Cauchy.

Thus, if $(X, U)$ is also complete, every net in $X$ has a convergent subnet, so $(X, U)$ is compact.

Columbia University

Received by the editors March 12, 1964. 